\title{
Role of Transient Elastography in Early Detection of Hepatocellular Carcinoma in Cirrhotic Patients
}

\author{
Hanaa A. Baddour, Hesham E. El Sheikh, Ahmed E. shalan
}

Department of radiology, Benha faculty of medicine, Benha University, Egypt.

Correspondence to

Hanaa A. Baddour Department of radiology, Benha faculty of medicine, Benha University, Egypt.

Email:

hermy_bloody@yahoo.com

Received: 26 August 2019

Accepted: 17 May 2021

\begin{abstract}
Background: Hepatocellular carcinoma is the most common primary malignancy of the liver and one of the most frequent causes of death in patients with liver cirrhosis. In Egypt it is one of the most challenging tumors with high incidence, prevalence and mortality rates. The degree of liver fibrosis is the strongest indicator of risk for HCC development that is why liver stiffness measured by TE is useful in demarcating patients at a high risk for $\mathrm{HCC}$, who require frequent check-up by imaging examinations. Aim of work: The aim of this study was to study the role of US elastography (FibroScan) in early detection of HCC in cirrhotic patients in comparison with triphasic CT. Patient and methods: This study was conducted on 100 patients with liver cirrhosis, 50 of them had HCC. The entire patients were diagnosed with liver cirrhosis by US examination and underwent fibroscan to estimate the degree of fibrosis and then triphasic CT to confirm or exclude the presence of HCC. Results: There is a correlation between high TE results and the incidence
\end{abstract} of HCC detection by CT, HCC detection was (100\%) in the groups with LSM value (>30KPa) and low $(9.6 \%)$ in the group with LSM value $(15-25 \mathrm{KPa})$. Conclusion: The higher LSM, the more liability for the patient to have HCC on CT scanning.

Keywords: Hepatocellular carcinoma, transient elastography, triphasic CT, liver stiffness measurement.

\section{Introduction}

HCC is a primary malignancy of the hepatocyte, the major cell type in the liver (1) Ultrasonography is the least expensive choice for screening, but it is highly operator-dependent. A suspicious lesion on a sonogram generally requires additional imaging studies to confirm the diagnosis and the stage of the tumor (2). The elasticity of a body is defined as the ability of the body to deform itself under the action of a mechanical force. 
The elasticity of a tissue can be estimated on the basis of the speed of propagation of wave. The higher the speed of propagation of that wave, the higher the stiffness of the tissue. Transient elastography (TE) measures such speed of propagation in relatively homogenous organs such as the liver, by using ultrasound (US) pulses to localize the shear elastic wave at different times (3).

\section{Patients and methods}

This prospective study was conducted between February 2018 to March 2019 on 100 patients with cirrhosis who visited Gastrointestinal Surgery Center of Mansoura University and gave their informed consent after approval of the institutional ethical committee. Fifty patients had cirrhosis with HCC diagnosed by US and confirmed by triphasic CT study and patients 50without evidence of HCC diagnosed by clinical, laboratory, US and confirmed by triphasic CT study.

The diagnosis of liver cirrhosis was done by US examination, then we performed fibroscan to these patients, either these patients had cirrhosis on US examination or not. Then triphasic CT was done to confirm liver cirrhosis and the presence of HCC.
Transient Elastography Liver stiffness measrment (LSM) was performed using Fibroscan, General Electric LOGIQ E9 (LE9) scanner with 2D shear wave elastography on a conventional ultrasound scanner. A time aligned sequential tracking (TAST) technique that enables high pulse repetition frequency (PRF) shear wave tracking on conventional ultrasound scanners was implemented with the Comb-push Ultrasound Shear Elastography (CUSE) technique on the General Electric LOGIQ E9 (LE9) scanner and combined to realize large field-of-view (FOV) 2D shear wave elastography. The elastic wave propagates through the underlying tissues the stiffer the tissue, the faster the shear wave propagates.

The median value of ten successful acquisitions expressed in kilopascal $(\mathrm{KPa})$ and was kept as representative of liver stiffness measurement. The clinical interpretation of TE depends on two important parameters for results to be considered reliable:

- The success rate (the ratio of the number of successful measurements to the total number of acquisitions) should be at least $60 \%$.

- IQR/Median (the coordination between different measurements) 
- Results were expressed in kiloPascals $(\mathrm{KPa})$ and 10 validated measurements were recorded for each patient (4).

The degree of fibrosis measured by TE correlated with the presence of HCC or not by triphasic CT scan.

liver stiffness measured noninvasively by TE has been reported to be well correlated with histologically assessed liver fibrosis stage (5).

\section{Results}

There was no statistical significant difference between the groups as

regards the age, sex or smoking; however HCC tends to be more common in males than females. Also, HCC tends to be more common in urban areas than rural areas and more common in farmers than non-farmers.

Hard liver was significantly common in HCC group. There is no statistical Most of cases were Okuda stage III, CLIP stage II, VISUM stage I and advanced Tokyo staging.

There was no statistical significant difference between the groups as regards the severity of liver cirrhosis assessed by MELD and UMELD scores however; they tend to be higher in HCC group. difference between the two groups as regards the frequency of hepatomegaly or splenomegaly.

Platelet count, RBCs count and albumin were significantly lower in HCC group. ALT, AST, ALP, INR and serum bilirubin were significantly higher in HCC group. Prothrombin time was significantly prolonged in HCC group.

There was no statistical significant difference between the groups as regard fasting blood sugar, hemoglobin, WBCs or s. creatinine; however s. creatinine tends to be higher in HCC group.

All studied patients of both groups were $\mathrm{HCV} \mathrm{Ab}$. positive and none of them were HBsAg positive.

Alpha fetoprotein levels were significantly higher in HCC group.

Most of patients of HCC group were Child C rather than Child A \& B.

All patients were cirrhotic; most of them had shrunken liver $(42 \%)$ and splenomegaly (94\%). Ascites was found in $22 \%, 34 \%$ had portal hypertension, $8 \%$ had esophageal varices and only $14 \%$ had portal vein thrombosis.

PVT was found in $10 \%$ of patients. Focal lesions by $\mathrm{CT}$ tend to be single, more in 
Right lobe, $\geq 5 \mathrm{~cm}$ in diameter. $10 \%$ of patients had enlarged lymph nodes and only $6 \%$ with hepatic vein involvement.

CT was superior in detection of both lymph nodes and hepatic vein involvement.

Liver stiffness and Inter Quartile Range measured by Fibroscan were significantly higher in HCC group. But, there was no statistical significant difference between the groups as regard the success rate (Fig1).

There is a correlation between high TE results and the incidence of $\mathrm{HCC}$ detection by $\mathrm{CT}$, HCC detection was $(100 \%)$ in the groups with LSM value $(>30 \mathrm{KPa})$ and low $(9.6 \%)$ in the group with LSM value (15-25KPa) (Fig 2)

It was found that likelihood of HCC risk was correlated with increase of liver stiffness. At liver stiffness of $<25 \mathrm{KPa}$, the probability of $\mathrm{HCC}$ presence was about $72 \%$ while stiffness of $25-30 \mathrm{KPa}$ has a probability of $91 \%$ so, these patients should undergo close follow up.

Patients with stiffness $\geq 30 \mathrm{KPa}$ had HCC.

There was significant positive correlation between liver stiffness by fibroscan and Child - Pugh, tumour size, Okuda classification, CLIP staging and Tokyo classification among HCC patients.

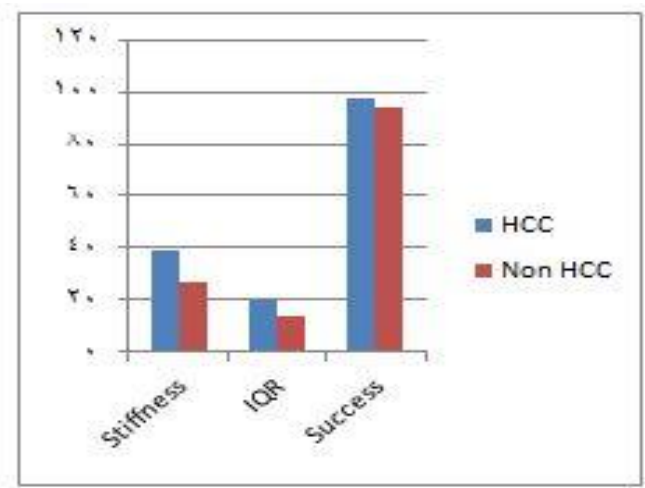

Figure (1) Fibroscan results of the studied patients.

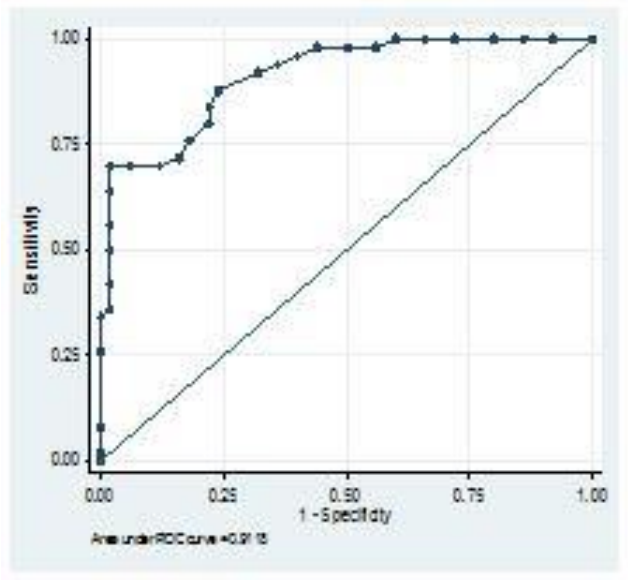

Figure ( 2 ) ROC curve analysis of Stiffness in prediction of hepatocellular carcinoma.

\section{Discussion}

In this study 100 patients were included, 50 patients with liver cirrhosis and did not had HCC, and 50 patients were cirrhotic and had HCC, patients with HCC had a mean age of 53.5 years with a range between 44-68 years old, while cirrhotic non-HCC patients are younger 
with a mean age of 51.3 years and ranging between 39-67 years old (6).

HCC commonly presented in males more than females with a male to female ratio $5.2: 1(7)$ and $58 \%$ of HCC cases were from urban areas (8)

The current study showed that $56 \%$ of HCC cases had a history of smoking (9). There was a traditional conflict about the link between cigarette smoking and the occurrence of HCC (10). But recent evidence support that smoking is a clear co-factor (11). In HCC patients, hepatomegaly and hard liver were observed in many patients (12).

Most of manifestations of decompensated liver cirrhosis such as ascites, jaundice, lower limb edema, hepatic encephalopathy that are common findings in chronic liver disease without HCC, are not useful in early suspicion or diagnosis of HCC comparing to cirrhosis (13).

AFP is the most commonly used biomarker for patients at risk for HCC (14). AFP in HCC cases had a mean value of $627.6 \mathrm{ng} / \mathrm{ml}$ which was statistically higher than that of patients with cirrhosis $11.7 \mathrm{ng} / \mathrm{ml}$.

Most of the patients with HCC (64\%) were Child C, followed by Child B (20\%) then Child A (16\%) (9).
According to MELD and uMELD scores, in this study the mean scores for patients with HCC were 20.6 and 4.2 respectively, while the mean scores for patients with cirrhosis were $\quad 19.9$ and 4.08 respectively(15).

US is very effective in early diagnosis of HCC as it can detect about $76 \%$ of early HCC patients (16). It is a good tool for HCC detection with a sensitivity of about $80 \%$ and specificity of over $90 \%$. Abdominal US was done to evaluate the liver status in the studied patients and all of the patients (100\%) with HCC had sonographic evidence of liver cirrhosis. By US examination, five patients (10\%) in this study were found to have portal vein thrombosis.

Twenty six patients, representing $52 \%$ of HCC cases had single focal hepatic lesion, while two focal lesions and multiple hepatic focal lesions were present in eight (16\%) and sixteen (32\%) of cases respectively (17).

In our study we observed that $18(36 \%)$ of HCC patients had their tumour size less than $5 \mathrm{~cm}$ while 32 (64\%) had their tumour size more than $5 \mathrm{~cm}$ (15), while the right lobe was predominantly more affected by tumour $(54 \%)$ than left lobe (10\%) and both lobes (36\%) (18). Five 
patients $(10 \%)$ of HCC cases were found to have lymph node enlargement (19).

As regard Okuda staging, most of $\mathrm{HCC}$ cases were in the late stage; stage III (46\%), followed by stage II (38\%) then stage I (16\%) (20).

According to CLIP staging, most of HCC cases were presented at the intermediate stage II $(62 \%)$ comparing to the patients presented at the early stage (10\%) and the advanced stage (28\%) respectively (20).

According to Tokyo staging, most of HCC patients were presented at advanced stage (58\%) comparing to the patients presented at early stage (42\%) (21).

The important observation in our study is that most of patients are diagnosed at advanced stages so; there must be a screening program for early detection of the patients with HCC.

When we used ROC curve, the cutoff value for $\mathrm{HCC}$ was $30.4 \mathrm{KPa}$ with sensitivity of about $72 \%$, specificity about $84 \%$, positive predictive value about $81.82 \%$ and negative predictive value about $75 \%(22)$.

There was a positive correlation between the liver stiffness \& the presence of HCC. Also, prediction of occurrence of HCC by fibroscan may be of useful value. SSLR for HCC presence by liver stiffness was
0.7272 in $<25 \mathrm{KPa}, 0.9167$ in 25.1 to $30 \mathrm{kPa}, 1.00$ in 30.1 to $35 \mathrm{KPa}, 1.1428$ in 35.1 to $40 \mathrm{kPa}$ and 1.3333 in $>40 \mathrm{KPa}$, so there is a direct correlation between high degree of LSM by TE and detection of HCC in cirrhotic patient by triphasic CT. Patients who had high LSM> $30 \mathrm{KPa}$ was proved $(100 \%)$ to had HCC by CT, while patients with LSM $<25 \mathrm{KPa}$ was found to had HCC on CT scanning with a percentage $72 \%$ and almost all the patients in our study that did not had HCC on CT scanning had LSM between $15-25$ (23).

Also there was direct correlation between the size of the tumor and stiffness measured by fibroscan. The bigger the size of the tumor; the higher the stiffness (24).

We noticed that there is a direct correlation between Okuda classification, CLIP staging and Tokyo classification with the stiffness measured by fibroscan. The more advanced liver disease (according to each classification); the higher stiffness of fibroscan.

In spite that liver biopsy is the gold standard diagnostic tool to compare with in our study for detection of hepatic focal lesions and incertitude about their nature rather than triphasic $\mathrm{CT}$, its' invasive painful interventional nature was a 
limitation that stimulated the search for noninvasive approaches but we recommend using biopsy as a gold standard modality in spite of triphasic CT to confirm the diagnosis of $\mathrm{HCC}$ for its certainty.

Our study was conducted on a small sample volume, so it needs to be applied on a large sample volume to be more reliable.

The final diagnosis was done using triphasic CT not biopsy as a gold standard diagnostic tool, which is known to be the most confirmative for diagnosis.

\section{Conclusion}

The results show that the higher LSM the more liability for the patient to have HCC on CT scanning.

Also there was direct correlation between the size of the tumor and stiffness measured by fibroscan. The bigger the size of the tumor; the higher the stiffness.

\section{References:}

1. Motola-Kuba, D.; Zamora-Valdés, D.; Uribe, M. (2006). Hepatocellular carcinoma.An overview. Ann. Hepatol.; 5 (1): 16-24.

2. B. Saar, F. Kellner-Weldon (2008) Radiological diagnosis of hepatocellular carcinoma. Liver Int. 2008 Feb; 28(2): 189199. doi: 10.1111/j.1478-3231.2007.01655.x
3. Sandrin, L.;Fourquet, B.;Hasquenoph,J.M.,S.Yon, C.Fournieret al. 2003): Transient elastography: a new noninvasive method for assessment of hepatic fibrosis. Ultrasound Med Biol.;29(12):170513.

4. Mohamed S Elzawawy, Shaimaa A Hassanein, Rasha M El Nomrosy (2018): The role of fibroscan in assessment of liver cirrhosis in patients with chronic liver disease. Menoufia medical journal, vol31,issue2

5. Castera, L.; Vergniol, J.; Foucher, J, BrigitteLe Bail, Chanteloup E., HaaserM., Darriet,P. Couzigou,V. Lédinghen et al.(2005): Prospective comparison of transient elastography, Fibrotest, APRI, and liver biopsy for the assessment of fibrosis in chronic hepatitis. C. Gastroenterology. 2005;128:34350.

6. El-Awady MK, Mostafa L, Tabll AA, Abdelhafez TH, Bader El Din NG, Zayed N, Shenawy RE, El Abd Y, Hasan RM, Zaghlol H, El Khayat H, Abdel Aziz AO. et al. (2012): Association of IL28B SNP With Progression of Egyptian HCV Genotype 4 Patients to End Stage Liver Disease. Hepat Mon. 2012 Apr;12(4):271-7. doi: 10.5812/hepatmon.835. Epub 2012 Apr 30. PubMed PMID: 22690235; PubMed Central PMCID: PMC3360937.

7. Alsina, A.E.; Beharry, A.; Beharry, N.Kemmer,E.Franco,H.Rojas,Guy W. Neff et al. (2012): Epidemiology of Hepatocellular Carcinoma in Florida - Part I: A Statewide Report. Florida Public Health Review; 9: 1823.

8. Attalla, M.S.; El-Azab, M.S.; El-Bakary, A.A. et al. (2009): IS AFLATOXIN B1 A COMMON RISK FACTOR FOR HEPATOCELLULAR CARCINOMA? 
Mansoura J. Forensic Med. Clin.Toxicol. Vol. XVII, No. 2.15-25

9. Abu El Makarem, M.A.; Abdel-Aleem, A.; Ali, A. Rafet Saber, M.Shatat. Reham DA.et al. (2011): Diagnostic significance of plasma osteopontin in hepatitis $\mathrm{C}$ virus related hepatocellular carcinoma. Ann Hepatol.; 10: 296-305.

10. El-Serag, H.B,; Richardson, P.A. and Everhart, J.E. ( 2001):The role of diabetes in hepatocellularcarcinoma: a case-control study among United States Veterans. Am. J. Gastroenterol.;96:2462-2467.

11. Trichopoulos, Christina Bamia (2011) Hepatocellular Carcinoma Risk Factors and Disease Burden in a European J Natl Cancer Inst.2011 Nov 16; 103(22): 1686-1695. doi: 10.1093ljncildjr395

12. Di Bisceglie, A.M. (2002): Epidemiology and clinical presentation of hepatocellular carcinoma. J Vasc Intery Radiol. 2002 Sep; 13(9 Pt): S169-71. PMID: 12354833

13. Mohamad, N.H.; Heba, M. E.; Nadia, M. M. et al. (2000): Review of epidemiologic and clinicopathologic features of 403 hepatocellular carcinoma (HCC) patients. Journal of the Egyptian Nat. Cancer Inst.; 12(2): 87-93.

14. T. Behne and M. S. Copur (2012): Biomarkers for Hepatocellular Carcinoma. Hindawi Publishing Corporation International Journal of Hepatology Volume 2012, Article ID 859076, 7 pages doi:10.1155/2012/859076.

15. Mohamed, A.A.; El-Toukhy, N.; Atta, M.M. (2013): Glypican-3 as a tumor marker for hepatocellular carcinoma. Journal of Applied Pharmaceutical Science;3 (06):083-087.

16. Zoli, M.; Magalotti, D.; Bianchi, G., Emilio Pisi, Cristina Gueli et al. (2007): Efficacy of asurveillance program for early detection of hepatocellular Carcinoma.Cancer; 78:977-85.

17. Abdelgawad IA, Mossallam GI, Radwan NH, ElZawahry HM, Elhifnawy NM (2013): Can Glypican3 be Diagnostic for Early Hepatocellular Carcinoma among Egyptian Patients? APJCP.;14(12):7345-7349.

18. Salem, R.; Gilbertsen, M.; Butt, Z. Memon;K. Vouche;M. Cella;D. et al. (2013 ):Increased quality of life among hepatocellular carcinoma patients treated with radioembolization, compared with chemoembolization.ClinGastroenterolHepatol. 2013 Oct;11(10):1358-1365

19. Gomaa, A.I.; Hashim, M.S. and Waked, I. (2014): Survival in Patients with Hepatocellular Carcinoma in Egypt. PLOS ONE | www.plosone.org . | Volume 9 | Issue 3 | e90929.

20. El-Zayadi, A.R.; Badran, H.M. Shawky, S., Emara;s, El-Bareedy;A. Sobhi;M.et al. (2010): Effect of surveillance for hepatocellular carcinoma on tumor staging and treatment decisions in Egyptian patients. Hepatol. Int.; 4(2):500-506.

21. Sarma, S.; Sharma, B.; Chawla, Y. K., Kapil;s., Singla; B., Kalra;N et al. (2010): Comparison of 7 staging systems in north Indian cohort of hepatocellular carcinoma.Tropical Gastroenterology;31(4):271-278.

22. Foucher, J.; Castéra, L.; Bernard, P.H. Adhoute, Xavier,et al. (2006 Prevalence and factors associated with failure of liver stiffness measurement using FibroScan in a prospective study of 2114 examinations. Eur J Gastroenterol Hepatol.;18(4):411-412.

23. Masuzaki, R.; Tateishi, R.; Yoshida (2009):Prospective risk assessment for hepatocellular carcinoma development in 
patients with chronic hepatitisC by transient elastography. Hepatology; 49: 1954-1961

24.Liana Gheorghe, SperantaIacob, CarmenGhidu, Iacob;R., Cerban;R., Croitoru;A., et al.,(2012):Liver Stiffness Value Assessed by Transient Elastography Correlates

With Nodule Size in Patients With Hepatocellular Carcinoma. Gastroenterology 142(5):S-979-S-980.

To cite this article: Hanaa A. Baddour, Hesham E. El Sheikh, Ahmed E. shalan. Role of Transient Elastography in Early Detection of Hepatocellular Carcinoma in Cirrhotic Patients. BMFJ 2021; 38(2):741-749. DOI: 10.21608/bmfj.2021.16278.1041 
